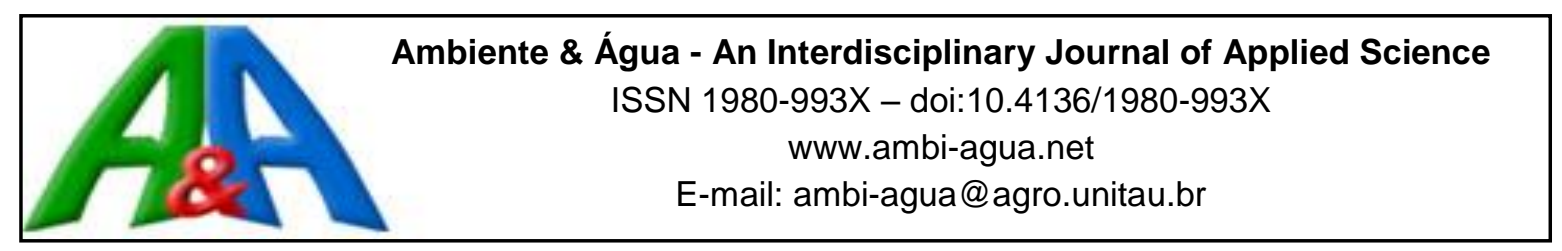

\title{
Remoción de plomo en soluciones acuosas empleando nanoaluminofosfatos amorfos
}

\author{
doi: 10.4136/ambi-agua.1437
}

Received: 24 Jun. 2014; Accepted: 25 Sep. 2014

\author{
Wendy Rondón $^{1}$; Ángela B. Sifontes ${ }^{1 *}$; David Freire ${ }^{1}$; Manuelita Quintal ${ }^{1}$; \\ Edgar Cañizales ${ }^{2}$; Franklin J. Méndez ${ }^{1}$; \\ Ligia Llovera ${ }^{1}$; Yraida Diaz ${ }^{1}$; Joaquin L. Brito ${ }^{1}$ \\ ${ }^{1}$ Instituto Venezolano de Investigaciones Científicas (IVIC), Caracas, Venezuela \\ Centro de Química \\ ${ }^{2}$ Instituto de Tecnología Venezolana para el Petróleo (Intevep), Caracas, Venezuela \\ Petróleos de Venezuela, S.A. (PDVSA) \\ "Autor correspondiente: e-mail: asifonte@ivic.gob.ve, \\ wrondon@ivic.gob.ve,dfreire6@gmail.com, manolis45@yahoo.com, \\ canizalesej@pdvsa.com,fmendez@ivic.gob.ve, 1llovera@ivic.gob.ve, \\ ygdiaz@ivic.gob.ve, joabrito@ivic.gob.ve
}

\section{RESUMEN}

Se emplearon nanopartículas de aluminofosfatos amorfos entre 23 a $30 \mathrm{~nm}$ de diámetro para la adsorción de iones plomo $\left(\mathrm{Pb}^{2+}\right)$ en soluciones acuosas. Los materiales fueron sintetizados mediante biosíntesis utilizando como plantillas biológicas las células de levadura Saccharomyces cerevisiae. Los estudios de adsorción se llevaron a cabo empleando el método batch, utilizando $100 \mathrm{mg}$ del sólido nanométrico y $200 \mu \mathrm{g} \mathrm{\textrm {L } ^ { - 1 }}$ de iones plomo. La determinación cuantitativa de $\mathrm{Pb}^{+2}$ fue realizada mediante espectrometría de absorción atómica con atomización electrotérmica (EAA-HG). Los valores obtenidos para el porcentaje de remoción (\% EF), capacidad de adsorción $\mathrm{q}_{\mathrm{e}}\left(\mu \mathrm{g} \mathrm{g}^{-1}\right)$ y coeficiente de remoción $\mathrm{K}_{\mathrm{d}}\left(\mathrm{mL} \mathrm{g}^{-1}\right)$ fueron de 40; 90 y 800 en promedio, respectivamente. Los resultados obtenidos demostraron las potencialidades que presentan estos materiales para la adsorción de iones $\mathrm{Pb}^{2+}$, siendo excelentes candidatos para la remediación de aguas contaminadas.

Palabras clave: adsorción, biosíntesis, nanopartículas.

\section{Remoção de chumbo em soluções aquosas usando nanoaluminofosfatos amorfos}

\section{RESUMO}

Empregaram-se nanopartículas de aluminofosfatos amorfos, com diâmetro de 23 a $30 \mathrm{~nm}$, para adsorção de íons chumbo $\left(\mathrm{Pb}^{2+}\right)$ em soluções aquosas. Os materiais foram preparados por meio de biossíntese usando como modelos biológicos Saccharomyces cerevisiae. Os estudos de adsorção foram feitos empregando-se o método batch, usando 100 mg do sólido nanométrico e $200 \mu \mathrm{g} \mathrm{L}^{-1}$ de íons chumbo. A determinação quantitativa de $\mathrm{Pb}^{2+}$ foi realizada por meio de espectrometria de absorção atômica com atomização eletrotérmica (EAA-HG). Os valores obtidos para a percentagem de remoção (\% EF), 
capacidade de adsorção $\mathrm{q}_{\mathrm{e}}\left(\mu \mathrm{g} \mathrm{g}^{-1}\right)$ e grau de remoção $\mathrm{K}_{\mathrm{d}}\left(\mathrm{mL} \mathrm{g}^{-1}\right)$ foram de 40; 90 e $800 \mathrm{em}$ média, respectivamente. Os resultados obtidos demonstraram as potencialidades destes materiais para a adsorção de íons $\mathrm{Pb}^{2+}$ e para a remediação de águas poluídas.

Palavras-chave: adsorção, biossíntese, nanopartículas.

\title{
Removal of lead in aqueous solutions using amorphous nanoaluminophosphates
}

\begin{abstract}
Amorphous aluminophosphates nanoparticles with diameter sizes from 23 to $30 \mathrm{~nm}$ were used for the adsorption of lead ions $\left(\mathrm{Pb}^{2+}\right)$ in aqueous solutions. The materials were biosynthesized using Saccharomyces cerevisiae as a biological template. Adsorption studies were conducted using the batch method, employing $100 \mathrm{mg}$ of the solid and $200 \mu \mathrm{g} \mathrm{L}^{-1}$ of lead ions. The quantitative determination of $\mathrm{Pb}^{2+}$ was carried out by electrothermal atomic absorption spectrometry (ETAAS). The percentage of removal (EF), adsorption capacity $\mathrm{q}_{\mathrm{e}}\left(\mu \mathrm{g} \mathrm{g}^{-1}\right)$ and removal coefficient $\mathrm{Kd}\left(\mathrm{mL} \mathrm{g}^{-1}\right)$ averaged 40, 90 and 800 , respectively. The results showed the potential of these materials for the adsorption of $\mathrm{Pb}^{2+}$ ions, and that these materials are good candidates for use in the remediation of contaminated water.
\end{abstract}

Keywords: adsorption, biosynthesis, nanoparticles.

\section{INTRODUCCIÓN}

En la actualidad, la contaminación por metales pesados en aguas subterráneas y superficiales, generada principalmente por actividades antropogénicas, representa una materia importante en el campo de la salud pública y el ambiente. Estas sustancias pueden ser causantes de graves daños en los seres vivos y son capaces de permanecer por largo tiempo en el entorno, circulando o acumulándose en sedimentos o incorporándose a la cadena alimenticia (Gupta et al., 1997; Sousa et al., 2010). La remoción de estos contaminantes por medio de procesos como el intercambio iónico y la adsorción, son considerados hoy en día como una alternativa eficiente. Sin embargo, en estos procesos se emplean materiales cuyo uso puede resultar prohibitivo debido a los altos costos asociados a su adquisición, implementación y operación (Gupta et al., 1997; Sousa et al., 2010). En este sentido, son muchas las investigaciones que se han desarrollado hacia la búsqueda de adsorbentes "no convencionales", de bajo costo y ambientalmente amigables (Gupta et al., 1997; Sousa et al., 2010). Por otra parte, en el área de purificación de aguas, la nanotecnología y la química verde están explorando nuevos campos asociados a la extracción eficiente de contaminantes y gérmenes. Las nanopartículas, nanomembranas y otros materiales nanoestructurados, están siendo utilizados para la detección y remoción de sustancias químicas y biológicas, incluyendo metales pesados (ejemplos: cadmio, cobre, plomo mercurio, níquel y cinc) (Tiwari et al., 2008). Básicamente cuatro tipos de materiales a escala nanométrica están siendo evaluados como materiales funcionales para aplicaciones en la purificación de aguas: nanopartículas que contienen metales, nanopartículas carbonáceas, zeolitas y dendrímeros (Tiwari et al., 2008). A este respecto, debido a su alta área superficial (relación superficie/volumen), los materiales a escala nanométrica exhiben mejores resultados en comparación con los adsorbentes convencionales, ofreciendo hoy en día nuevas perspectivas para su aplicación en los procesos de purificación de aguas (Gupta et al., 2011; Jia et al., 2009; Tiwari et al., 2008). En este particular, recientemente ha sido investigada la remoción de metales pesados en aguas empleando nanopartículas de aluminofosfatos mesoporosos 
amorfos. Estos materiales han demostrado excelente capacidad de adsorción para cationes de $\mathrm{Hg}, \mathrm{Cd}$, As y Fe (Das et al., 2012). No obstante, no han sido realizados estudios sobre la adsorción de iones plomo.

Los efectos tóxicos del plomo son bien conocidos y la máxima concentración aceptada, para el agua de consumo humano, está fijada en todos los países del mundo a niveles muy bajos $\left(\leq 50 \mu \mathrm{g} \mathrm{L}^{-1}\right)$ (Tiwari et al., 2008). Entre otras consideraciones, la contaminación con este elemento puede ocurrir cuando el agua fluye a través de tuberías que contienen plomo en su composición (Sublet et al., 2003). Es por ello, que la presente contribución exhibe un estudio preliminar a fin de explorar las propiedades que presentan los nanoaluminofosfatos amorfos en la remoción de iones plomo en medio acuoso. El adsorbente utilizado fue preparado mediante biosíntesis, empleando levadura, Saccharomyces cerevisiae, como plantilla biológica para la formación de la estructura inorgánica (Sifontes et al., 2013). Es importante destacar que esta nueva ruta de síntesis emplea materiales que presentan alta biodegrabilidad, escasa toxicidad y bajo costo (Sifontes et al., 2013).

\section{MATERIALES Y MÉTODOS}

Los nanoaluminofosfatos empleados en este estudio se sintetizaron mediante un procedimiento publicado en la literatura por Sifontes et al. (2013). El tamaño de partícula fue estimado por microscopía electrónica de transmisión, determinándose un rango entre 23 y $30 \mathrm{~nm}$ (Sifontes et al., 2013). El material biológico utilizado correspondió a células de levadura de tipo comercial. Todos los reactivos empleados fueron de grado analítico. Los sólidos preparados se caracterizaron utilizando un difractómetro de rayos-X (DRX) Siemens modelo D-5000, empleando radiación $\mathrm{CuK} \alpha$ en el rango $2 \theta$ entre $5^{\circ}$ y $70^{\circ}$ a fin de examinar su posible estructura cristalina. El área superficial se determinó por adsorción de nitrógeno a $-196{ }^{\circ} \mathrm{C}$, en un equipo Micromeritics ASAP 2010, empleando el método de BET. Las observaciones mediante microscopía electrónica de barrido (MEB-EDX) fueron realizadas en un instrumento HITACHI S-2400. Los espectros de infrarrojo con Transformada de Fourier (FTIR) se obtuvieron empleando un equipo Perkin Elmer Spectrom RX1. Los estudios por RMN de estado sólido (RMN-MAS de ${ }^{27} \mathrm{Al} \mathrm{y}{ }^{31} \mathrm{P}$ ) se realizaron en un espectrómetro Bruker Avance $300 \mathrm{MHz}$. Para la síntesis, procedimientos analíticos y los estudios de adsorción se empleó agua ultrapura $(18 \mathrm{M} \Omega \cdot \mathrm{cm})$ obtenida con un equipo desionizador marca Millipore modelo Milli-Q (Millipore Corporation). Los patrones acuosos necesarios para la cuantificación fueron preparados por dilución de las soluciones metálicas estándar de 1000 $\mathrm{mgL}^{-1}$ (Merck). Un equipo Varian Techtron modelo AA-875 de doble haz equipado con un horno de grafito GTA-95 (EAA-HG) fue requerido para la determinación de plomo. Las mediciones de $\mathrm{Al}$ y $\mathrm{P}$ se realizaron con un espectrómetro de emisión atómica con plasma inductivamente acoplado (ICP-OES), OPTIMA 3000 (Perkin Elmer).

\subsection{Estudio de adsorción}

Se estudió el efecto de captación de plomo en medio acuoso empleando los nanomateriales sintetizados previamente. Las medidas de adsorción se realizaron mediante la técnica de muestras por lotes (batch) a temperatura ambiente $\left(20 \pm 1^{\circ} \mathrm{C}\right)$. La cantidad de metal removido por gramo de adsorbente (capacidad de adsorción $\mathrm{q}_{\mathrm{e}}\left(\mu \mathrm{g} \mathrm{g}^{-1}\right)$ ) se evaluó mediante el estudio de tres muestras representativas de los materiales sintetizados (Singh et al., 2011). A tal fin, $100 \mathrm{mg}$ de cada muestra adsorbente fueron añadidos a $100 \mathrm{~mL}$ de una solución de 200 $\mu \mathrm{g} \mathrm{L}^{-1}$ de $\mathrm{Pb}^{2+}$ preparada a partir de una solución patrón de $1000 \mu \mathrm{g} \mathrm{L}^{-1}$ de $\mathrm{Pb}^{2+}$. La mezcla fue colocada en un agitador de tipo vortex $(1500 \mathrm{rpm})$ por periodos de 5 y $24 \mathrm{~h}$ para determinar el efecto del tiempo de contacto con el material adsorbente. Posteriormente cada solución fue centrifugada durante $1 \mathrm{~min}$ y el sobrenadante obtenido fue filtrado y 
diluido 5 veces. Se consideró como blanco (muestra control), una muestra del material sintetizado sin levadura, la cual fue sometida al mismo tratamiento de adsorción que los nanomateriales en estudio. La determinación de plomo en la solución final se realizó empleando EAA-HG. Se emplearon soluciones estándares para calibrar el instrumento. El valor de $\mathrm{q}_{\mathrm{e}}\left(\mu \mathrm{g} \mathrm{g}^{-1}\right)$ se determinó según la Ecuación 1 (Singh et al., 2011; Sprynskyy et al., 2006).

$$
q_{e}=\frac{\left(\begin{array}{ll}
C_{i} & C_{f}
\end{array}\right) V_{s o l}}{m_{a d s}}
$$

donde:

$\mathrm{C}_{\mathrm{i}} \mathrm{y}_{\mathrm{f}}$ son las concentraciones inicial y final en equilibrio, de los iones metálicos en la solución en $\mu \mathrm{g} \mathrm{L}^{-1}$,

$\mathrm{V}_{\text {sol }}$ es el volumen de la solución en litros y

$\mathrm{m}_{\mathrm{ads}}$ es la masa del adsorbente en gramos.

Adicionalmente, también se calculó la constante de adsortividad o coeficiente de distribución $\mathrm{K}_{\mathrm{d}}\left(\mathrm{mL} \mathrm{g}^{-1}\right)$ relacionada con la selectividad y afinidad de la adsorción y el porcentaje de eficiencia o remoción \% EF, empleando las Ecuaciones 2 y 3, para todos los nanomateriales sintetizados con levadura y para el blanco (muestra control) (Singh et al., 2011; Sprynskyy et al., 2006).

$$
\begin{aligned}
& K_{d}=\frac{q_{e}}{C_{f}} \\
& \% E F=\frac{K_{d} m_{a d s}}{\left(\begin{array}{ll}
K_{d} & m_{a d s}+V_{s o l}
\end{array}\right)} 100
\end{aligned}
$$

\section{RESULTADOS Y DISCUSIÓN}

\subsection{Caracterización del material adsorbente}

Se realizó un estudio por DRX para verificar la presencia o ausencia de fases cristalinas en los sólidos sintetizados. La Figura 1 presenta los difractogramas para el blanco y la muestra preparada mediante biosíntesis. En ellos puede observarse una banda ancha en el rango $2 \theta$ de $15-40^{\circ}$, la cual es atribuida a la naturaleza amorfa de los materiales sintetizados (Liu et al., 2006a; Sifontes et al., 2013).

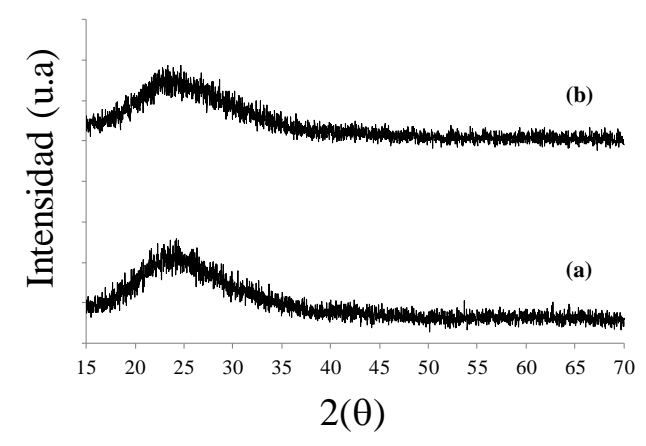

Figura 1. DRX de los aluminofosfatos sintetizados sin levadura (a) con levadura (b). 
En la Figura 2 es mostrado el espectro de ${ }^{27}$ Al-RMN-MAS correspondiente a los aluminofosfatos sintetizados con y sin la presencia de levadura. En la Tabla 1 se recopilan los valores de los desplazamientos químicos correspondientes.

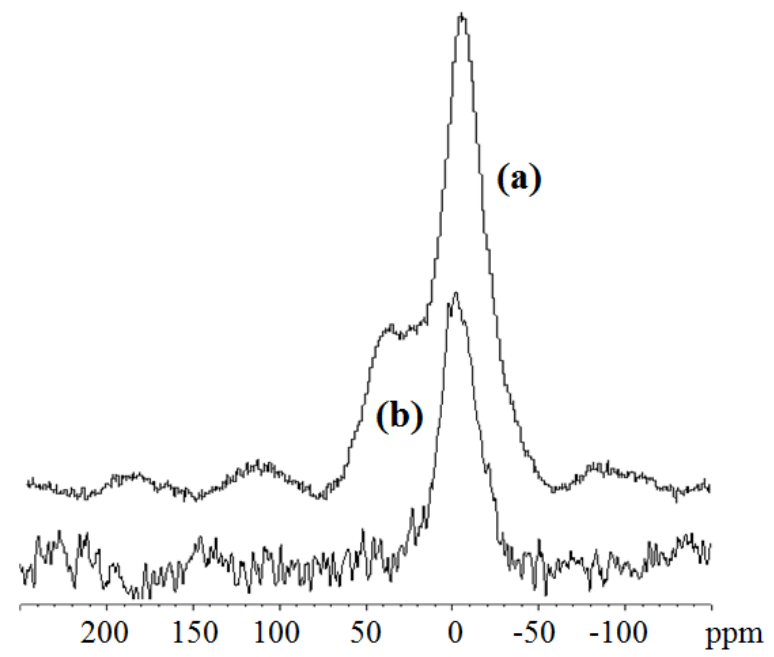

Figura 2. Espectro de ${ }^{27} \mathrm{Al}-\mathrm{RMN}-\mathrm{MAS}$ de los aluminofosfatos sintetizados sin levadura (a), con levadura (b).

La inexistencia de $\mathrm{Al}^{3+}$ en coordinación tetraédrica pareciera sugerir ausencia de material de tipo aluminofosfato, ya que como se indicó antes, la señal correspondiente a $\mathrm{Al}^{3+}$ tetraédrico es asignada a grupos $\left[\mathrm{Al}(\mathrm{PO})_{4}\right]$. Sin embargo, la existencia de cationes de aluminio en una coordinación diferente de cuatro ya ha sido postulada para los sistemas AlPO amorfos (Marquez et al., 1997). En este particular varios investigadores proponen que estos materiales amorfos están constituidos por una fase rica en alúmina, con disminución del contenido de fósforo $(\mathrm{Al} / \mathrm{P}>1)$ y que estos sistemas $\mathrm{AlPO}_{4}-\mathrm{Al}_{2} \mathrm{O}_{3}$ son construidos a partir de unidades de tetraedros $\left[\mathrm{PO}_{4}\right]$ y poliedros de $\left[\mathrm{AlO}_{4}\right],\left[\mathrm{AlO}_{5}\right]$ y $\left[\mathrm{AlO}_{6}\right]$ (Liu et al., 2006a; Marquez et al., 1997).

Tabla 1. Resumen de los desplazamientos químicos obtenidos mediante los análisis de ${ }^{27} \mathrm{Al}-\mathrm{RMN}-\mathrm{MAS}$ y ${ }^{31} \mathrm{P}-\mathrm{RMN}-\mathrm{MAS}$ para los AlPOs sintetizados.

\begin{tabular}{lcc}
\hline \multirow{2}{*}{ Muestra } & \multicolumn{2}{c}{$\begin{array}{c}\text { Desplazamiento químico } \\
(\mathbf{p p m})\end{array}$} \\
\cline { 2 - 3 } & ${ }^{27} \mathbf{A l}$ & ${ }^{31} \mathbf{P}$ \\
\hline \multirow{3}{*}{ AlPO Control } & 38,67 & 29,94 \\
& & $-10,45$ \\
& $-5,52$ & $-47,05$ \\
\hline \multirow{2}{*}{ AlPO sintetizado } & 2,30 & 28,04 \\
con levadura & $-2,30$ & $-16,16$ \\
& $-6,90$ & $-56,56$ \\
\hline
\end{tabular}

Esto pudiera dar soporte a los resultados obtenidos mediante ${ }^{27} \mathrm{Al}-\mathrm{RMN}-\mathrm{MAS}$. En este sentido, adicionalmente fue efectuada la determinación de la relación de Al/P mediante los estudios realizados por ICP-OES. El valor promedio obtenido fue $\mathrm{Al} / \mathrm{P}=1,6$, lo cual demuestra, en concordancia con lo anteriormente descrito, un enriquecimiento de aluminio 
para los AlPO obtenidos mediante biosíntesis. Por otra parte, se ha encontrado que las especies de fosfatos asociadas con átomos de aluminio generalmente presentan desplazamientos químicos ${ }^{31} \mathrm{P}$ en el rango de -7 a $-30 \mathrm{ppm}$, dependiendo del grado de cristalinidad, contenido de agua y grado de condensación (Duffy e VanLoon, 1995; Liu et al., 2006b; Marquez et al., 1997). Las señales ${ }^{31} \mathrm{P}$ con desplazamiento químico a campo bajo, de 0 a -13 ppm, pueden ser asignadas a las especies de $\mathrm{P}$ con muy bajo grado de condensación (Figura 3).

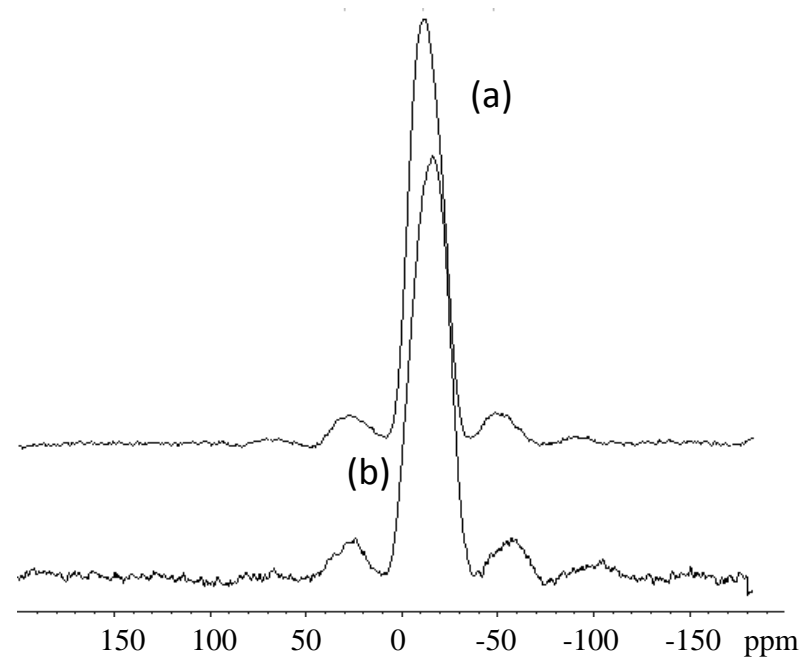

Figura 3. Espectro de ${ }^{31} \mathrm{P}-\mathrm{RMN}-\mathrm{MAS}$ de los aluminofosfatos sintetizados sin levadura (a), con levadura (b).

En los espectros de ${ }^{31}$ P-RMN-MAS mostrados en la Figura 3, se asignaron los desplazamientos químicos correspondientes al sólido sintetizado y la muestra control. Las señales identificadas se atribuyen a la presencia de especies de $\mathrm{P}$ en coordinación tetraédrica con enlaces $\mathrm{O}-\mathrm{Al}, \mathrm{P}(\mathrm{OAl})_{4}$. La señal de resonancia relativamente ancha podría implicar la presencia de pequeñas cantidades de especies de $\mathrm{P}$ parcialmente coordinadas con grupos $\mathrm{OH}$ o $\mathrm{H}_{2} \mathrm{O}$ como por ejemplo $\mathrm{P}(\mathrm{OAl})_{\mathrm{x}}(\mathrm{OH})_{4-\mathrm{x}}$ (Duffy e VanLoon, 1995; Liu et al., 2006b; Marquez et al., 1997). Así mismo, en la Tabla 1 son mostrados los valores de los desplazamientos químicos obtenidos del espectro de ${ }^{31} \mathrm{P}-\mathrm{RMN}-\mathrm{MAS}$, tanto para la muestra control como para la preparada con levaduras. En la Figura 4 se muestra el espectro FTIR correspondiente a los sólidos sintetizados con y sin levadura. Mediante este estudio se pudo confirmar la presencia de los grupos funcionales característicos de los aluminofosfatos, entre los cuales se pudieron determinar las señales más importantes. En las regiones alrededor de $3600 \mathrm{y} \mathrm{de} 1600 \mathrm{~cm}^{-1}$ se encuentran unas bandas atribuidas al estiramiento y flexión del enlace O-H de la molécula de agua (Kannan et al., 2013; Liu et al., 2006a; 2006b). Entre 1422-959 $\mathrm{cm}^{-1}$ fue observada una banda de fuerte intensidad, la cual se corresponde con las vibraciones de tensión P-O de los grupos $\mathrm{PO}_{4}{ }^{3-}$ (Kannan et al., 2013; Liu, et al., 2006a; 2006b). Del mismo modo, fueron asignadas las bandas ubicadas en la región de $712-469 \mathrm{~cm}^{-1}$ a las vibraciones simétricas de tensión P-O-Al. Una señal de moderada intensidad a $560 \mathrm{~cm}^{-1}$ fue atribuida a las vibraciones de flexión O-P-O de los grupos $\left(\mathrm{PO}_{4}\right)^{3-}$ (Kannan et al., 2013; Liu, et al., 2006a; 2006b). La ausencia de las bandas en la región $2000-2950 \mathrm{~cm}^{-1}$ para las dos muestras sintetizadas, las cuales se atribuyen a las vibraciones simétricas y asimétricas de los grupos $\mathrm{C}-\mathrm{H}$, indican la eliminación completa de la plantilla biológica (levadura).

En las Figuras 5 y 6 se presentan las micrografías (MEB) de las células de levadura antes y después del tratamiento de síntesis. Adicionalmente, es presentado un análisis por EDX el cual permite evidenciar claramente la naturaleza de los aluminofostatos sintetizados. 


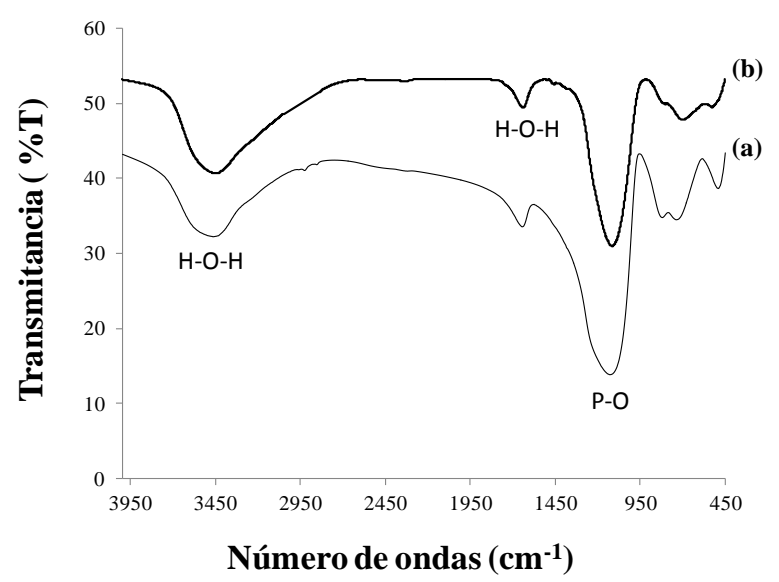

Figura 4. Espectro IR de los aluminofosfatos sintetizados sin levadura (a) y con levadura (b).

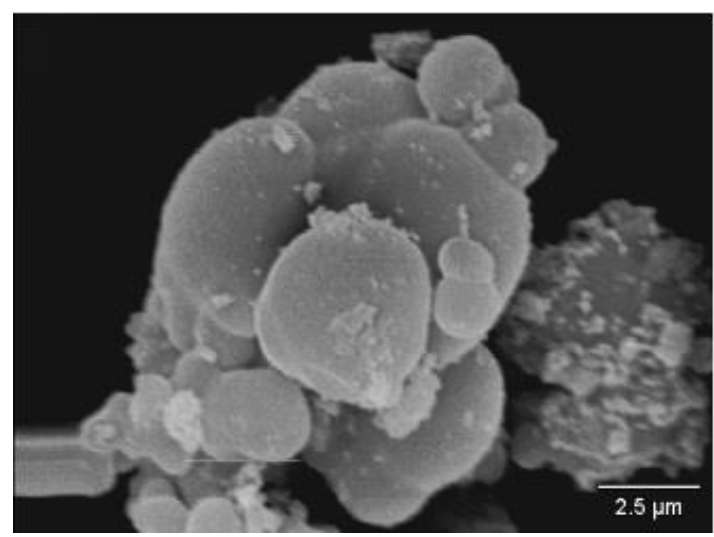

Figura 5. Imagen SEM de células de levadura de Saccharomyces cerevisiae.

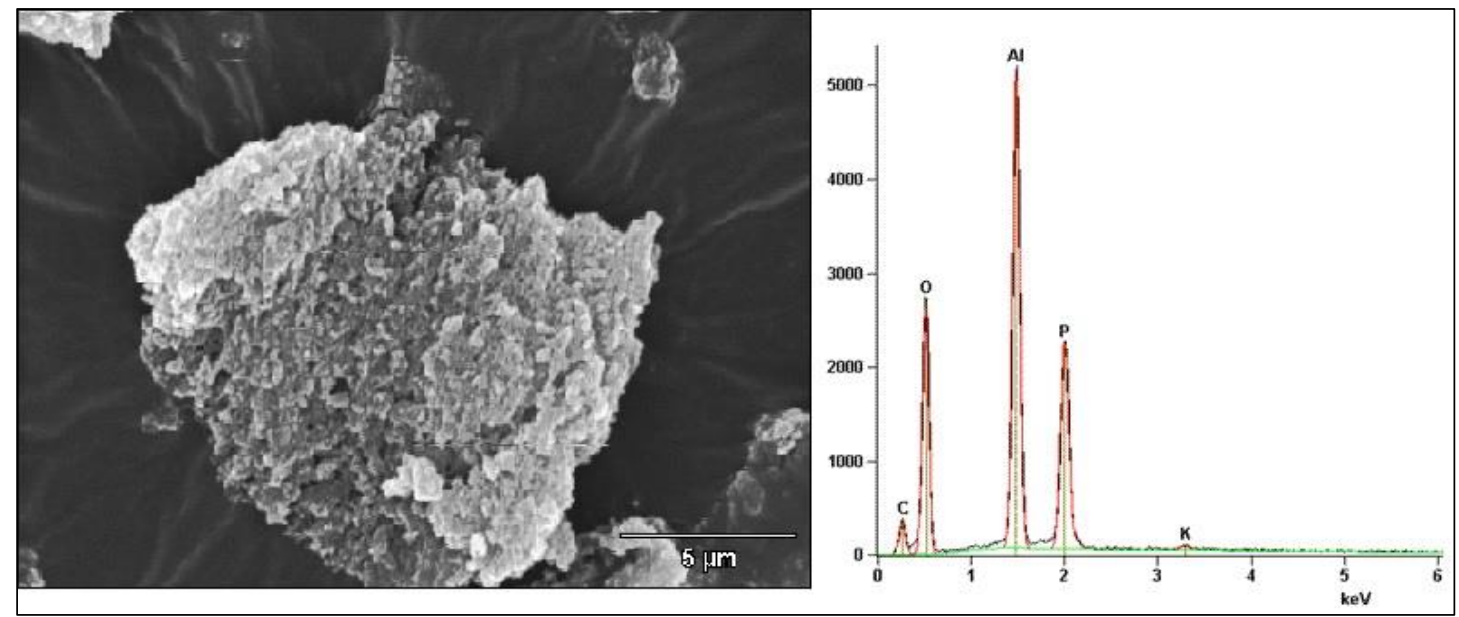

Figura 6. Panel izquierdo: Imagen SEM de las células de levadura Saccharomyces cerevisiae después del tratamiento de síntesis. Panel derecho: Espectro EDX de las células de levadura después del tratamiento de síntesis.

La Figura 7 muestra las isotermas resultantes del estudio por adsorción física de $\mathrm{N}_{2}$ para la muestra sintetizada con los microorganismos y la muestra control, calcinada a $650{ }^{\circ} \mathrm{C}$. Las isotermas observadas presentan un comportamiento tipo IV (Barrett et al., 1951) con una abertura de histéresis desplazada a altas presiones. Esto puede ser atribuido a la presencia de una mesoporosidad extra-estructural generada por los espacios ubicados entre los agregados 
de nanopartículas. En cuanto al volumen total de poros $\left(\mathrm{V}_{\mathrm{t}}\right)$, las muestras exhibieron valores que indican porosidad media con diámetros de poro promedios entre 10 y $20 \mathrm{~nm}$. En la Tabla 2 se muestran los valores de área superficial, diámetro y volumen de poro para los sólidos sintetizados, apreciándose las diferencias entre el material sintetizado y la muestra control.

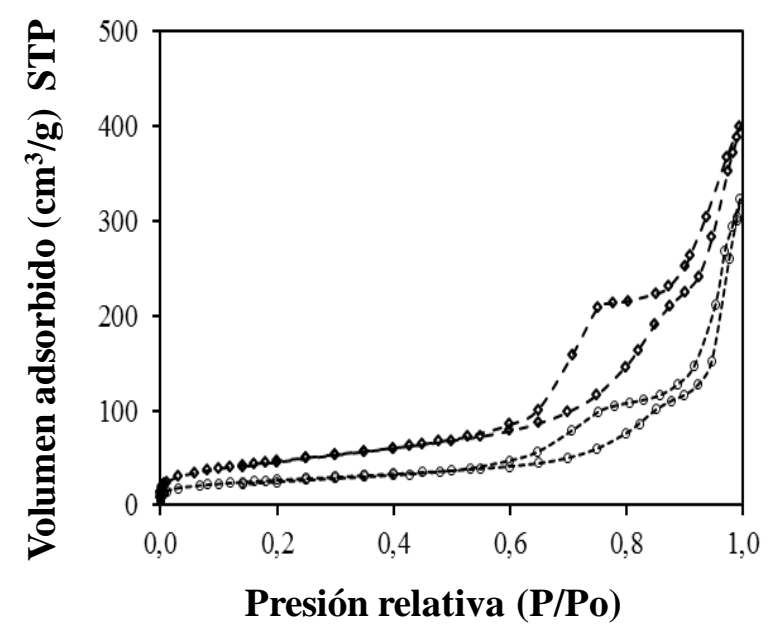

Figura 7. Isotermas de adsorción física de $\mathrm{N}_{2}$ para la muestra Control -o- y la sintetizada con los microorganismos $-\diamond-$.

Tabla 2. Área superficial y tamaños de los poros de las muestras.

\begin{tabular}{lccccc}
\hline Muestra & $\mathbf{S}_{\text {BET }}\left(\mathrm{m}^{2} \mathrm{~g}^{-1}\right)$ & $\mathbf{S}_{\text {micro }}\left(\mathrm{m}^{2} \mathrm{~g}^{-1}\right)$ & $\mathbf{S}_{\text {meso }}\left(\mathrm{m}^{2} \mathrm{~g}^{-1}\right)$ & $\mathbf{V}_{\mathbf{t}}\left(\mathrm{cm}^{3} \mathrm{~g}^{-1}\right)$ & Dp $(\mathrm{nm})$ \\
\hline Control & 93 & 13 & 80 & 0,5 & 17 \\
M & 164 & 0 & 164 & 0,6 & 13 \\
\hline
\end{tabular}

Nota: $\mathrm{S}$ : área superficial; $\mathrm{V}_{\mathrm{t}}$ : volumen total de poros; Dp: diámetro de poros, $\mathrm{M}$ : promedio de las muestras M1 y M2.

La porosidad observada en las muestras tratadas con las células de levadura es atribuida a la acción de las biomoléculas presentes en sus membranas celulares, las cuales pueden actuar diferentemente favoreciendo la fijación de precursores y actuando como centros de nucleación (Merroun et al., 2005). Estas contienen grupos funcionales, tales como grupos amino y carboxilato (entre otros), capaces de interaccionar con los precursores metálicos introducidos en el lecho de síntesis (Sifontes et al., 2013). La superficie de las células de levadura adquiere una carga eléctrica, debido a la ionización de estos grupos químicos superficiales lo cual le permitirá interactuar con los iones o moléculas presentes en el medio exterior (Sifontes et al., 2013; Pompe et al., 2013).

\subsection{Estudios de adsorción}

Debido a que recientemente ha sido investigada con éxito la remoción de metales pesados en aguas, empleando nanopartículas de aluminofosfatos mesoporosos amorfos (Gupta et al., 1997; Sousa et al., 2010) y dadas las ventajosas características estructurales y texturales observadas en los materiales sintetizados empleando levadura como plantilla biológica, fue considerado de particular interés, realizar algunos estudios sobre la adsorción de iones metálicos en medio acuoso. Esto permitiría evaluar las potencialidades de los nanoaluminofosfatos amorfos preparados mediante biosíntesis como material adsorbente. Para ello, se realizó la determinación de plomo por EAA-HG, en solución acuosa (Tabla 3) y se evaluaron los parámetros de adsorción, \% EF, qe y $\mathrm{K}_{\mathrm{d}}$ para ambos materiales (Tabla 4). Para 
verificar la dispersión experimental, se emplearon dos muestras separadas de material sintetizado por biosíntesis, M1 y M2 (réplicas).

Tabla 3. Determinación de la adsorción de iones de plomo en $100 \mathrm{mg}$ de aluminofosfatos.

\begin{tabular}{lcc}
\hline Adsorbentes & $\begin{array}{c}\text { Horas de } \\
\text { contacto }\end{array}$ & $\begin{array}{c}\text { Concentración } \mathbf{P b}^{\mathbf{2 +}} \\
\left(\boldsymbol{\mu g} \mathbf{~ L}^{-1}\right)^{*}\end{array}$ \\
\hline M1 & 5 & 117,84 \\
M2 & 5 & 104,77 \\
Control & 5 & 140,56 \\
M1 & 24 & 54,21 \\
M2 & 24 & 68,41 \\
Control & 24 & 109,88 \\
\hline
\end{tabular}

Nota: M1 y M2: muestras sintetizadas con levadura, réplicas. Control: muestra sintetizada sin levadura. La concentración inicial de $\mathrm{Pb}^{2+}$ fue de $200 \mu \mathrm{g} \mathrm{L}{ }^{-1}$ en todos los experimentos.

"Calculado utilizando la curva de calibración lineal con ecuación de regresión lineal $\mathrm{Abs}=0,0040 \mathrm{C}+0,0120$ y $\mathrm{R}^{2}=0,9989$ obtenida con el EAA-HG.

Los resultados obtenidos mediante la determinación de plomo en solución acuosa permitieron estimar el porcentaje de eficiencia o remoción de los materiales adsorbentes. El sólido preparado mediante biosíntesis presentó una mayor eficiencia de remoción que la muestra control, preparada sin levadura. Los valores oscilaron entre 40-48 \% para 5 horas y de $65-73 \%$ para $24 \mathrm{~h}$ de exposición. Los valores obtenidos empleando la muestra control fueron de 30 y $45 \%$ para 5 y $24 \mathrm{~h}$ de exposición, respectivamente. Esto puede ser atribuido a la mayor área superficial que presentan los materiales preparados con levadura (ver Tabla 2). Se calculó el valor promedio de los parámetros de adsorción experimentales $\mathrm{q}_{\mathrm{e}} \mathrm{y} \mathrm{K}_{\mathrm{d}}$ obtenidos para las muestras de los materiales preparados mediante biosíntesis. Los resultados mostrados son los siguientes: $\mathrm{q}_{\mathrm{e}}(88,69 \pm 9,24) \mu \mathrm{g} \mathrm{g}^{-1}$ y K $\mathrm{d}(803,00 \pm 149,62) \mathrm{mL} \mathrm{g}^{-1}$ correspondientes para 5 horas de contacto y de $\mathrm{q}_{\mathrm{e}}(138,69 \pm 10,05) \mu \mathrm{g} \mathrm{g}^{-1}$ y $\mathrm{K}_{\mathrm{d}}(2306,66 \pm 541,74) \mathrm{mL} \mathrm{g}^{-1}$ para 24 horas de contacto. En la Tabla 5, se comparan estos resultados con los obtenidos por otros autores. Si bien es cierto que los materiales sintetizados no alcanzan una eficiencia del $90 \%$ para la remoción de $\mathrm{Pb}^{+2}$, ofrecen sin embargo, una alternativa interesante la cual es económica, viable y menos contaminante que las vías de síntesis convencionales. Los estudios realizados sugieren que las aplicaciones de estos materiales pudieran ser evaluadas también para otros iones metálicos como cobalto, zinc y cadmio.

Tabla 4. Parámetros de adsorción estimados para 100 mg de aluminofosfatos.

\begin{tabular}{lcccc}
\hline Adsorbentes & $\begin{array}{c}\text { Horas de } \\
\text { contacto }\end{array}$ & $\mathbf{q}_{\mathbf{e}}\left(\boldsymbol{\mu} \mathbf{g ~ g}^{\mathbf{- 1}}\right)$ & $\mathbf{K}_{\mathbf{d}}\left(\mathbf{m L ~ \mathbf { g } ^ { - \mathbf { 1 } } )}\right.$ & $\mathbf{\%} \mathbf{E F}$ \\
\hline M1 & 5 & 82,16 & 697,20 & 41,08 \\
M2 & 5 & 95,23 & 908,80 & 47,61 \\
Control & 5 & 59,43 & 422,80 & 29,72 \\
M1 & 24 & 145,80 & 2689,73 & 72,90 \\
M2 & 24 & 131,59 & 1923,59 & 65,80 \\
Control & 24 & 90,12 & 820,01 & 45,06 \\
\hline
\end{tabular}

Nota: M1 y M2: muestras sintetizadas con levadura, réplicas. Control: muestra sintetizada sin levadura. 
Tabla 5. Comparación de diferentes materiales adsorbentes empleados en la remoción de iones plomo.

\begin{tabular}{lccc}
\hline \multicolumn{1}{c}{ Material } & Dosis $(\mathbf{m g})$ & $\begin{array}{c}\text { Tiempo de } \\
\text { Contacto }(\mathbf{h})\end{array}$ & $\mathbf{q}\left(\mathbf{m g ~ g}^{\mathbf{- 1}}\right)$ \\
\hline Hongos Mucor rouxii & 50 & 7 & 35,69 \\
Fosfato natural & 100 & 3 & 115,34 \\
Fosfato activado & 100 & 3 & 155,04 \\
Magnetita/Hidroxiapatita & 50 & 24 & 598,80 \\
AlPO Control & 100 & 5 & 95,23 \\
AlPO sintetizado con levadura & 100 & 5 & 59,43 \\
\hline
\end{tabular}

\section{CONCLUSIONES}

Mediante el procedimiento de síntesis utilizado, se generó un material nanométrico de elevada porosidad (nanoaluminofosfatos amorfos), característica obtenida como producto de las interacciones entre la pared celular y el precursor inorgánico. Se realizaron los estudios de adsorción de iones plomo en soluciones acuosas obteniendo eficiencias entre 40 y $70 \%$, capacidad de adsorción entre 50 y $145 \mu \mathrm{g} \mathrm{g}^{-1}$ y una constante de distribución en el orden de los 400 a $2600 \mathrm{~mL} \mathrm{~g}^{-1}$. Estos resultados evidencian que los sólidos preparados mediante biosíntesis pueden ser empleados para la remoción de trazas de iones plomo y podrían ser aplicados como materiales adsorbentes de otros iones metálicos en soluciones acuosas.

\section{AGRADECIMIENTOS}

Los autores agradecen al Instituto Venezolano de Investigaciones Científicas (IVIC) por el apoyo a través de los proyecto No 1077 y No 1112 y al Laboratorio de Análisis Químico Estructural de Materiales de la Universidad de Los Andes (LAQUEM-ULA).

\section{REFERENCIAS}

BARRETT, E. P.; JOYNER, L. G.; HALENDA, P. P. The determination of pore volume and area distributions in porous substances. I. Computations from Nitrogen Isotherms. Journal of the American Chemical Society, v. 73, n. 1, p. 373-380, 1951. http://dx.doi.org/10.1021/ja01145a126

DAS, S. K.; BHUNIA, M. K.; BHAUMIK, A. Solvothermal synthesis of mesoporous aluminophosphate for polluted water remediation. Microporous and Mesoporous Materials, v. 155, p. 258-264, 2012.

http://dx.doi.org/10.1016/j.micromeso.2012.01.034

DUFFY, S. J.; VANLOON, G. W. Investigations of aluminum hydroxyphosphates and activated sludge by27Al and31P MAS NMR. Canadian Journal of Chemistry, v. 73, n. 10, p. 1645-1659, 1995. http://dx.doi.org/10.1139/v95-204

GUPTA, V. K.; AGARWAL, S.; SALEH, T. A. Synthesis and characterization of aluminacoated carbon nanotubes and their application for lead removal. Journal of hazardous materials, v. 185, n. 1, p. 17-23, 2011. http://dx.doi.org/10.1016/j.jhazmat.2010.08.053 
GUPTA, V. K.; SRIVASTAVA, S. K.; MOHAN, D.; SHARMA, S. Design parameters for fixed bed reactors of activated carbon developed from fertilizer waste for the removal of some heavy metal ions. Waste Management, v. 17, n. 8, p. 517-522, 1997. http://dx.doi.org/10.1016/s0956-053x(97)10062-9

JIA, K.; PAN, B.; LV, L.; ZHANG, Q.; WANG, X.; PAN, B.; ZHANG, W. Impregnating titanium phosphate nanoparticles onto a porous cation exchanger for enhanced lead removal from waters. Journal of Colloid and Interface Science, v. 331, n. 2, p. 453457, 2009. http://dx.doi.org/10.1016/j.jcis.2008.11.068

KANNAN, C.; MUTHURAJA, K.; DEVI, M. R. Hazardous dyes removal from aqueous solution over mesoporous aluminophosphate with textural porosity by adsorption. Journal of hazardous materials, v. 244-245, p. 10-20, 2013.

http://dx.doi.org/10.1016/j.jhazmat.2012.11.016

LIU, G.; JIA, M.; ZHOU, Z.; WANG, L.; ZHANG, W.; JIANG, D. Synthesis and pore formation study of amorphous mesoporous aluminophosphates in the presence of citric acid. Journal of Colloid and Interface Science, v. 302, n. 1, p. 278-286, 2006a. http://dx.doi.org/10.1016/j.jcis.2006.06.026

LIU, G.; WANG, Z.; JIA, M.; ZOU, X.; ZHU, X.; ZHANG, W. et al. Thermally stable amorphous mesoporous aluminophosphates with controllable P/Al ratio: synthesis, characterization, and catalytic performance for selective O-methylation of catechol. The Journal of Physical Chemistry B, v. 110, n. 34, p. 16953-16960, 2006b. http://dx.doi.org/10.1021/jp062824u

MARQUEZ, A. M.; OVIEDO, J.; SANZ, J. F.; BENITEZ, J. J.; ODRIOZOLA, J. A. Geometric and electronic structure of amorphous aluminophosphates. Ab initio and experimental studies. The Journal of Physical Chemistry B, v. 101, n. 46, p. 95109516, 1997. http://dx.doi.org/10.1021/Jp972147r

MERROUN, M. L.; RAFF, J.; ROSSBERG, A.; HENNIG, C.; REICH, T.; SELENSKAPOBELL, S. Complexation of Uranium by Cells and S-Layer Sheets of Bacillus sphaericus JG-A12. Applied and Environmental Microbiology, v. 71, p. 5532, 2005. http://dx.doi.org/10.1128/AEM.71.9.5532-5543.2005.

POMPE, W.; RÖDEL, G.; WEISS H-J.; MERTIG, M. Bio-nanomaterials, designing materials inspired by nature. [S.1.]: Wiley-VCH Verlag GmbH \& Co.KGaA, 2013.

SIFOnTES, Á. B., GONZÁleZ, G., TOVAR, L. M., MÉNDEZ, F. J., GOMES, M. E., CAÑIZALES, E. et al. Biosynthesis of amorphous mesoporous aluminophosphates using yeast cells as templates. Materials Research Bulletin, v. 48, n. 2, p. 730-738, 2013. http://dx.doi.org/10.1016/j.materresbull.2012.11.016

SINGH, B. K.; BHADAURIA, J.; TOMAR, R.; TOMAR, B. S. Effect of humic acid on sorption of trace metal ions by sodium aluminosilicate. Desalination, v. 268, n. 1-3, p. 189-194, 2011. http://dx.doi.org/10.1016/j.desal.2010.10.022

SOUSA, F. W.; OLIVEIRA, A. G.; RIBEIRO, J. P.; ROSA, M. F.; KEUKELEIRE, D.; NASCIMENTO, R. F. Green coconut shells applied as adsorbent for removal of toxic metal ions using fixed-bed column technology. Journal of Environmental Management, v. 91, n. 8, p. 1634-1640, 2010. http://dx.doi.org/10.1016/j.jenvman.2010.02.011 
SPRYNSKYY, M.; BUSZEWSKI, B.; TERZYK, A. P.; NAMIESNIK, J. Study of the selection mechanism of heavy metal $\left(\mathrm{Pb}^{2+}, \mathrm{Cu}^{2+}, \mathrm{Ni}^{2+}\right.$, and $\left.\mathrm{Cd}^{2+}\right)$ adsorption on clinoptilolite. Journal of Colloid and Interface Science, v. 304, n. 1, p. 21-28, 2006. http://dx.doi.org/10.1016/j.jcis.2006.07.068

SUBLET, R.; SIMONNOT, M. O.; BOIREAU, A.; SARDIN, M. Selection of an adsorbent for lead removal from drinking water by a point-of-use treatment device. Water Research, v. 37, n. 20, p. 4904-4912, 2003. http://dx.doi.org/10.1016/j.watres.2003.08.010

TIWARI, D. K.; BEHARI, J.; SEN, P. Application of nanoparticles in waste water treatment. World Applied Sciences Journal, v. 3, n. 3, p. 417-433, 2008. 